\title{
Echocardiographic assessment of left ventricular systolic function
}

\author{
Lars Gunnar Klaeboe ${ }^{1,2} \cdot$ Thor Edvardsen ${ }^{1,2}$
}

Received: 4 October 2018 / Accepted: 22 October 2018 / Published online: 2 November 2018

(c) The Author(s) 2018

\begin{abstract}
Left ventricular (LV) ejection fraction (LVEF) is the most validated and commonly used echocardiographic measure of systolic function. LVEF has a unique position in cardiology having severed as selection criteria for therapeutic trials that constitute the evidence base of today's treatment recommendations. Assessment of LV systolic function by global longitudinal strain (GLS) from speckle tracking echocardiography (STE) is a sensitive and feasible method that overcomes many of the limitations of LVEF, including reproducibility issues of serial testing and detection of LV dysfunction in pathologically remodeled hearts. This review discusses the role of STE as a complementary method to LVEF in estimation of LV systolic function.
\end{abstract}

Keywords Systolic function $\cdot$ Ejection fraction $\cdot$ Speckle tracking echocardiography $\cdot$ Global longitudinal strain

\section{Introduction}

Evidence of left ventricular (LV) systolic dysfunction has significant implications for diagnosis, risk assessment, and follow-up of patients with heart disease and evaluation of LV systolic function is the most common indication for echocardiography. There are currently several echocardiographic measures of LV function available, as methodology has moved from linear measurements, via two-dimensional (2D) echocardiography with volume estimation and regional and global deformation analysis, to three-dimensional (3D) echocardiography. Assessment of LV function is challenging even with better and more automated methods. Ideally, one wants a sensitive and accurate measurement of LV function that correlates with the patient's symptoms, provides important information about the course of illness and can guide therapy. There is, however, no such perfect parameter.

The different components of myocardial fiber architecture contributing to a normal systolic function are unequally affected by different cardiac diseases, making different

Thor Edvardsen

thor.edvardsen@medisin.uio.no

1 Department of Cardiology and Center for Cardiological Innovation, Oslo University Hospital, Rikshospitalet, PO Box 4950, 0424 Oslo, Norway

2 Faculty of Medicine, Institute of Clinical Medicine, University of Oslo, Oslo, Norway echocardiographic measures of LV systolic function more or less sensitive to detect subtle myocardial dysfunction. All the left ventricle function parameters measure contraction or volumes, and are therefore affected by loading conditions, which must be taken into account in every interpretation of LV function. The clinician needs to know the strengths and weaknesses of different LV functional parameters and master multiple methods, as patient-specific factors and clinical settings can make different echocardiographic techniques more or less appropriate at the time of examination. This review summarizes the most widely used echocardiographic methods for evaluating LV systolic function in clinical practice and discusses their strengths and weaknesses.

\section{The methodology and clinical use of echocardiographic ejection fraction}

The most widely used and accepted echocardiographic parameter of LV systolic function has been ejection fraction (EF), defined as the fraction of stroke volume and enddiastolic volume. This parameter has a unique position in cardiology, having served as a selection criterion for almost all landmark therapeutic trials of heart failure, and is well incorporated in clinical guidelines [1].

Echocardiographic LVEF was historically estimated based on the dimensions from linear methods by M-mode and 2D echocardiography, later by volume assessment-based 2D and 3D methodology. The geometrical assumptions 
underlying linear estimation of LVEF do not hold in several commonly encountered clinical settings, e.g., regional wall motion abnormalities and altered ventricular dimensions and geometry. The linear method is no longer recommended in routine clinical practice. The European Association of Cardiovascular Imaging (EACVI) and the American Society of Echocardiography (ASE) recommend assessment of LVEF in 2D by modified Simpson's method of discs, acquiring LV volumes from apical 4- and 2-chamber views, or alternatively, when available and feasible, by 3D-based full-volume acquisition [2].

\section{The limitations of LVEF}

Although LVEF as an index of LV systolic function has broad applications, it also has important shortcomings. Twodimensional LVEF estimations based on biplane Simpson's method of discs suffer from modest reproducibility, as the exact imaging planes are difficult to recapture. As a rule of thumb, 2D LVEF changes of less than 10 percentage points between examinations do not necessarily represent an actual change in systolic function [3]. This limits the usefulness of 2D LVEF in sequential follow-up of patients.

LVEF depends on good imaging quality for manual or automated tracking of endocardial border and is vulnerable to foreshortening. Calculating 2D LVEF by Simpson's method of discs is based on the assumption that the LV has an ellipsoid shape. Three-dimensional echocardiography overcomes this limitation using full-volume acquisition. While LVEF measured in 3D is more reproducible than 2D, it still depends on good image quality, and suffers from lower spatial and temporal resolution than 2D images in general. The higher temporal variability of 2D LVEF makes the semi-automated 3D LVEF with superior intra- and interobserver and test-retest variability suited for serial evaluation of LV systolic function [3].

Even under optimal imaging conditions, LVEF can be an incomplete or even an incorrect estimate of LV function. This is especially true in significant concentric remodeling, hypertrophic cardiomyopathy or small cavity size, where there is significant systolic dysfunction with reduced stroke volume despite a normal or supranormal LVEF. As recently highlighted, reduced myocardial deformation despite preserved LVEF can be explained through a change of geometric factors such as increased wall thickness and reduced end-diastolic volume [4]. Strain will better reflect systolic function in these patients with preserved EF due to geometric confounders.

Moreover, LVEF is dependent on current loading conditions and can be both over- and underestimated due to loading conditions. An example of this is the reduced preload in severe mitral regurgitation, which may cause increased
LVEF due to significant regurgitation volume. The opposite might be true in severe aortic stenosis and other states with markedly increased afterload. Bradycardia may cause an overestimation of LVEF due to increased stroke volume. Inversely, the reduced stroke volume in tachycardia or variable R-R interval of atrial fibrillation may lead to LVEF underestimation.

One final important limitation is the uncertain significance of near-normal LVEF measurements. Above a value of 40-45\%, LVEF does not correlate with symptoms, inform prognosis [5], or offer a guide to treatment, although significant systolic dysfunction still might be evident with more sensitive methods [6].

\section{Left ventricular systolic function assessed by myocardial deformation imaging}

Normal systolic function is a result of contraction of myocardial fibers arranged in a right-handed helix in the subendocardium, a left-handed helix in the subepicardium, and a layer of circumferential fibers in between. Briefly, the interaction of the double helical-oriented muscle layers causes longitudinal and circumferential shortening, and radial thickening, in combination with a twisting motion along the long axis in systole.

\section{Nomenclature of deformation imaging}

Myocardial deformation imaging by echocardiography involves assessment of displacement and velocities of cardiac tissue during systole and diastole. Strain is a dimensionless index of deformation, describing the percentage change in a myocardial segment length in the longitudinal, circumferential direction or radial direction relative to baseline. By convention, negative strain values are assigned to shortening and thinning, while strain values for lengthening and thickening are positive. Strain rate (SR) is the rate at which the deformation occurs, but is rarely used in clinics. LV rotational mechanics are described by the terms rotation, twist, and torsion. Rotation, expressed in degrees, describes the deformation angle measured in short axis. Positive values denote clockwise rotation and negative values denote counterclockwise rotation seen from the apex. Twist is the absolute difference in rotation between base and apex. Torsion is LV twist divided by the length of LV, allowing comparison of rotational mechanics between left ventricles of differing sizes. 


\section{Overview of myocardial deformation methodology}

Echocardiographic strain imaging was originally developed as an extension of tissue Doppler velocity imaging (TDI) by calculating strain rate (SR) based on the differences in velocities between two points in myocardium relative to the distances between the points measured. The utility of TDI-based measurements are limited to longitudinal strain due to the angle dependency of Doppler-based methods, and the technique is sensitive to poor signal-to-noise ratio.

Speckle-tracking echocardiography (STE) is based on the frame by frame tracking of the natural acoustic markers of gray scale ultrasound images, or so-called "speckles", through the cardiac cycle. The speckles are generated by the interaction between ultrasound waves and cardiac tissue, and can be tracked, independent of viewing angle, to produce segmental strain and strain rate curves in the longitudinal, circumferential, and radial direction of the LV. In contrast to the 1-dimensional transducer velocities of TDI strain, STE measures 2D displacement of speckles, making it possible to differentiate pathologically contracting segments from normal, and thus assess both regional and global myocardial function.

Validation against sonomicrometry and tagged magnetic resonance imaging has shown that STE provides accurate and angle-independent measurements of regional myocardial strain [7]. Initiation of the Strain Standardization Task Force and a consensus document for a common standard of myocardial deformation imaging [8] have made variability between different machine and software vendors lower in GLS than other conventional echocardiographic indices of LV systolic function. Circumferential and radial strains, segmental longitudinal strain and regional functional abnormalities $[9,10]$, however, still show greater intervendor differences and measurement variability not sufficiently reproducible for routine clinical work. As normal values of GLS depend on the position of measurement in the myocardium and vendor-specific factors, universal normal values are yet to be recommended, although a peak GLS of approximately $20 \pm 2 \%$ has been suggested as a guide to what can be expected in healthy persons [2].

3D STE offers simultaneous multi-directional deformation analysis, including assessment of rotational mechanics, using a full-volume acquisition of the $\mathrm{LV}$, and holds promise for the future of LV functional assessment. However, current 3DE scans generally have lower spatial and temporal resolutions than 2DE, and 3D STE is still regarded as an experimental method.

\section{Clinical application of speckle tracking echocardiography}

GLS has been shown to be a more sensitive marker of LV systolic function than LVEF, with superior prognostic value in various heart diseases with a wide range myocardial dysfunction [11]. This quantitative method for assessment of regional and global LV function has gained popularity in detecting left ventricular systolic dysfunction in cardiomyopathies, cardio-oncology, or in the presence of cardiac remodeling where LVEF is still within the normal range. Assessment of GLS provides additional information regarding heterogeneity and timing of ventricular contractions that has been shown to be prognostically important in several cardiac conditions. Furthermore, the visualization of disease-specific strain patterns and regional differences in myocardial function as qualitative bull's eye plots or polar maps has been clinically useful.

One must, however, recognize that STE echocardiography has its limitations. Notwithstanding comparable or better reproducibility than conventional echocardiographic indices, GLS is still dependent on good imaging quality, and is vulnerable to heart rate variability, breathing translation, and placement of region of interest in the myocardium. Furthermore, correct identification of cardiac events such as aortic valve closure to determine end-systole is essential. Group comparisons and serial follow-up of patients should currently be limited to the same equipment and segment model.

\section{Refinement of prognosis and risk assessment}

GLS is extensively evaluated and has shown to add information to LVEF in assessment of prognosis and event prediction [11, 12]. Importantly, GLS provides incremental prognostic information in patients with LVEF better than $35 \%$ regarding mortality, and is a stronger prognostic marker of all-cause mortality and the composite endpoint of cardiac death, hospitalization for heart failure and ventricular arrhythmias [11]. It is also important to acknowledge that LV systolic dysfunction is common in heart failure with preserved ejection fraction (HFpEF). Impaired GLS has been shown to be a risk marker of cardiac death and mortality in HFpEF independent of clinical predictors, LVEF and diastolic function [13].

Eligibility for primary prevention implantable cardiac defibrillator (ICD) and cardiac resynchronization therapy (CRT) is currently dependent on severely reduced LV systolic function, although ventricular arrhythmias are not uncommon in patients with LVEF better than $35 \%$, which is the current upper limit for primary prevention. Speckle tracking echocardiography has shown ability to identify patients with more preserved LVEF at higher risk of ventricular arrhythmias, although the current LVEF-guided strategy still applies. GLS is the strongest independent predictor of ventricular arrhythmias and also heart failure, and all-cause mortality in HCM with preserved LVEF [14]. Furthermore, assessment of the inter-segmental variability in contraction duration of longitudinal strain, mechanical dispersion, has 
shown to be an accurate marker of ventricular arrhythmias in channelopathies, ischemic heart disease, and dilated cardiomyopathy [15] and could provide future therapeutic advancement.

\section{Systolic function in cardiomyopathies and asymptomatic patients}

Assessing LV systolic function in the presence of pathological remodeling is a common challenge in clinical practice. In addition to excellent prognostic abilities, GLS provides more accurate assessment of systolic function in conditions with increased LV mass or altered LV geometry. This is particularly evident in hypertrophic cardiomyopathy (HCM) where a supranormal LVEF may be present in severely depressed systolic function. In HCM, the most impaired strain values correspond to areas of fibrosis and greatest wall thickness, with a typical pattern of impaired septal strain easily identified on bull's eye plots. The ESC guidelines for diagnosis and management include myocardial strain imaging in early HCM disease evaluation [16]. Furthermore, impaired longitudinal strain can be seen in mutation-positive family members who have not yet developed other phenotypic features of HCM [16].

The differential diagnosis of HCM vs increased wall thickness in other conditions such as athlete's heart, cardiac amyloidosis, and Fabry disease is challenging. A normal GLS measurement in an athlete makes physiological athletic remodeling more likely than underlying myocardial disease [17]. Additionally, bull's eye plots have shown to be clinically useful to visualize disease-specific strain patterns. Apical sparing (Fig. 1) is a regional strain pattern, highly sensitive and specific of cardiac amyloidosis, while isolated posterolateral defects are typical of Fabry disease [18].

Abnormal GLS in asymptomatic diabetic and hypertensive patients may be a marker of subclinical or latent LV systolic dysfunction, and confers elevated cardiovascular risk [19]. In asymptomatic severe aortic stenosis (AS), ASE/ESC guidelines recommended aortic valve replacement (AVR) when $\mathrm{LVEF}<50 \%$ [20]. LVEF, however, is not a sensitive measure of systolic dysfunction, particularly in the setting of the hypertrophy and fibrosis in the pressure-overloaded myocardium of AS. Furthermore, impaired LV longitudinal strain is a correlate of myocardial fibrosis in aortic stenosis, and is associated with worse functional recovery after AVR in symptomatic patients [21]. A GLS worse than $15 \%$ is associated with mortality and future symptom-driven AVR, independent of STS risk score and other imaging indices of AS severity in asymptomatic patients with preserved LVEF [22]. Although, not yet incorporated in societal guidelines, multimodal imaging recommendations suggest that the asymptomatic patients with severe AS, normal LVEF, and abnormal GLS are at high risk and should be considered for AVR. Moreover, abnormal GLS is a strong predictor of allcause mortality in patients with mild, moderate, and severe AS [23].

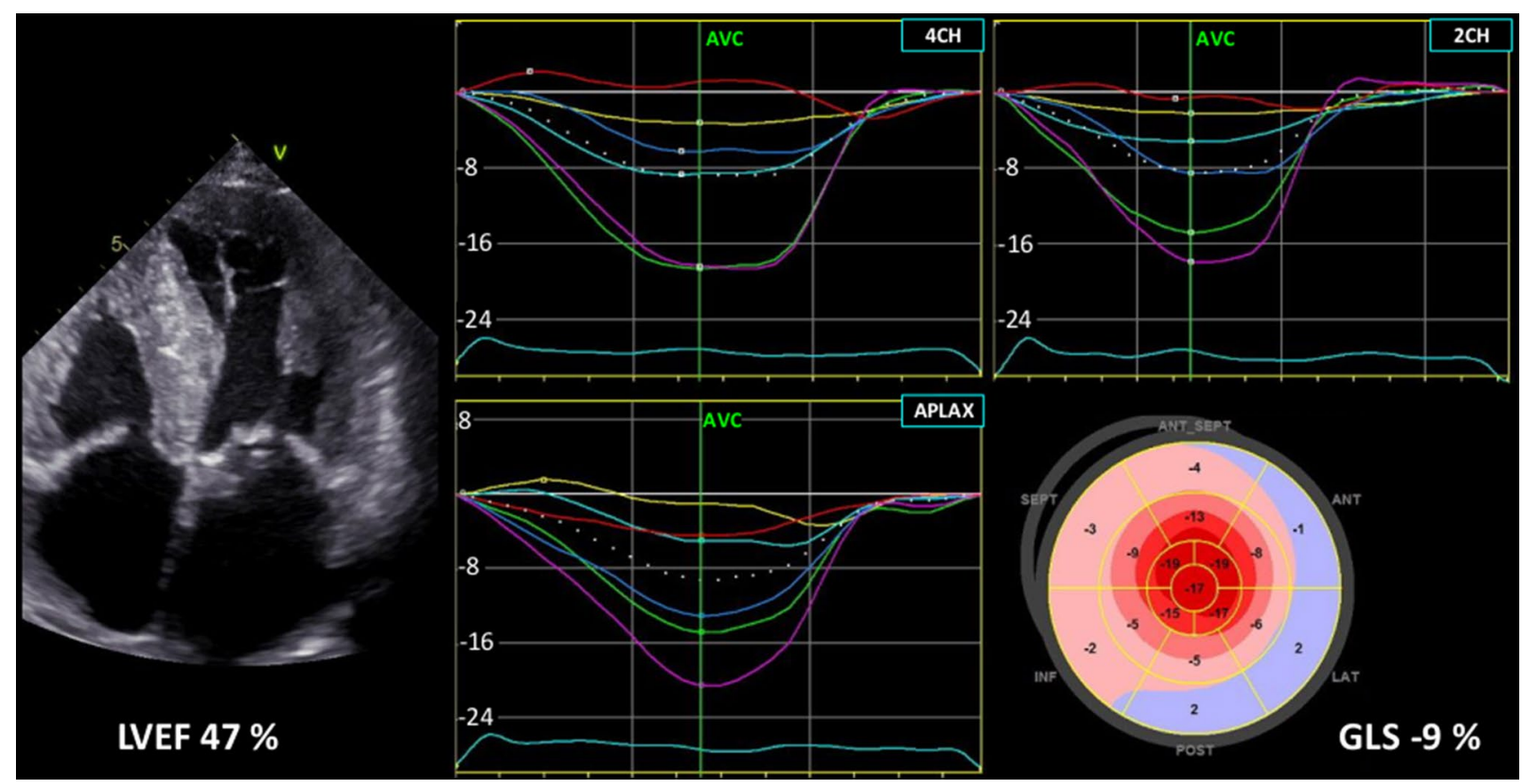

Fig. 1 Patient with amyloidosis, mildly impaired left ventricular ejection fraction (LVEF) and characteristic speckle tracking findings including apical sparing and severely depressed systolic function as indicated by abnormal global longitudinal strain (GLS) 
As mention earlier, LVEF is a poor measure of systolic function in severe mitral regurgitation (MR). This is reflected by current guidelines' use of a supranormal LVEF cutoff value of $60 \%$ as the lower limit of LV systolic performance below which surgical intervention is recommended in asymptomatic severe MR. Preoperative GLS worse than $18 \%$, however, has been shown to be strongly associated with postoperative reduction of $\mathrm{LVEF}$, irrespective of preoperative LVEF.

\section{Coronary artery disease}

The use of STE echocardiography has been well studied in patients with coronary artery disease (CAD) and is particularly useful in the acute setting when LVEF is normal or wall motion abnormalities are not visible. As the subendocardial longitudinal-oriented muscle fibers are vulnerable to ischemia, early systolic lengthening and postsystolic shortening [24] in adjacent segments or reduced strain corresponding to coronary supply territories are longitudinal strain features of ischemia (Fig. 2). A faster interventional strategy facilitated by strain imaging in myocardial infarction where ECG is not sensitive enough to detect coronary occlusion has been demonstrated to be beneficial [25]. Furthermore, GLS has been shown to be better than LVEF in prediction of all-cause mortality and infarct size after myocardial infarction [26].

\section{Cardio-oncology}

LV dysfunction is the limiting factor for the use of many anti-neoplastic agents. Cancer therapeutics-related cardiac dysfunction (CTRCD) has been defined as an asymptomatic decrease in LVEF of more than $10 \%$ to subnormal reference values $(\mathrm{LVEF}<53 \%)$ or a symptomatic decrease of more than $5 \%$ in LVEF following anti-neoplastic treatment, regardless of baseline values [27]. As decisions regarding cessation of potential lifesaving cancer therapy are based on the LVEF changes, it is of paramount importance that the measurements are accurate and show low variability over
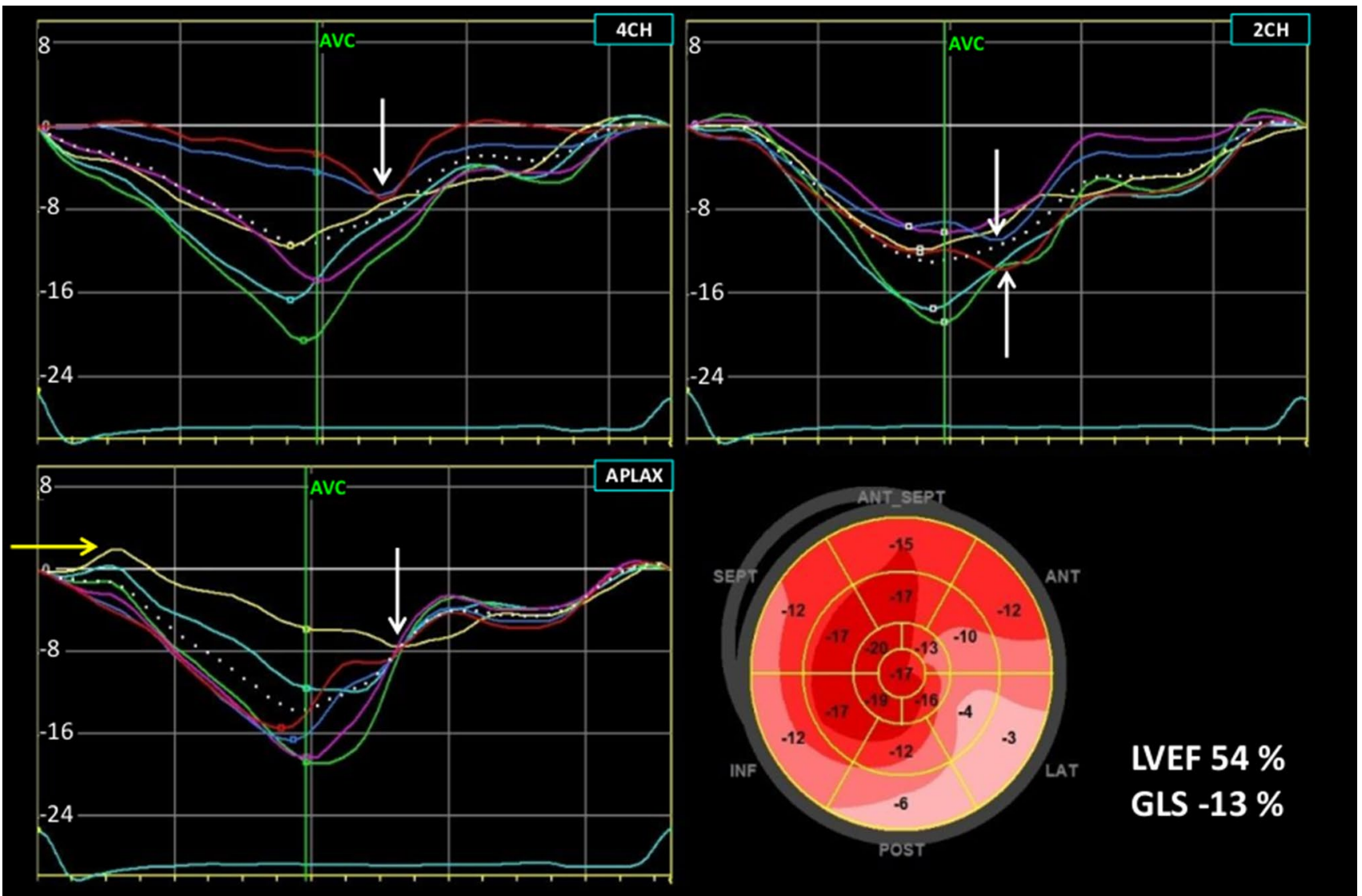

Fig. 2 Patient with significant stenosis on the circumflex and right coronary artery, and typical ischemic longitudinal strain features including a non-uniform reduction of longitudinal strain (bull's eye plot), early systolic lengthening (yellow arrow) and postsystolic

shortening (white arrows). Left ventricular (LV) systolic function is clearly reduced as shown by abnormal global longitudinal strain (GLS) despite preserved left ventricular ejection fraction (EF) 


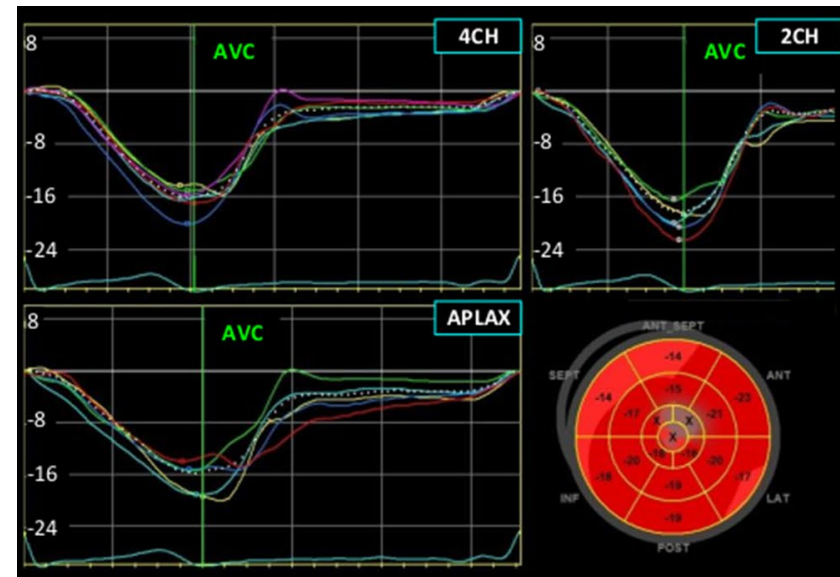

LVEF $50 \%$, GLS -18\%

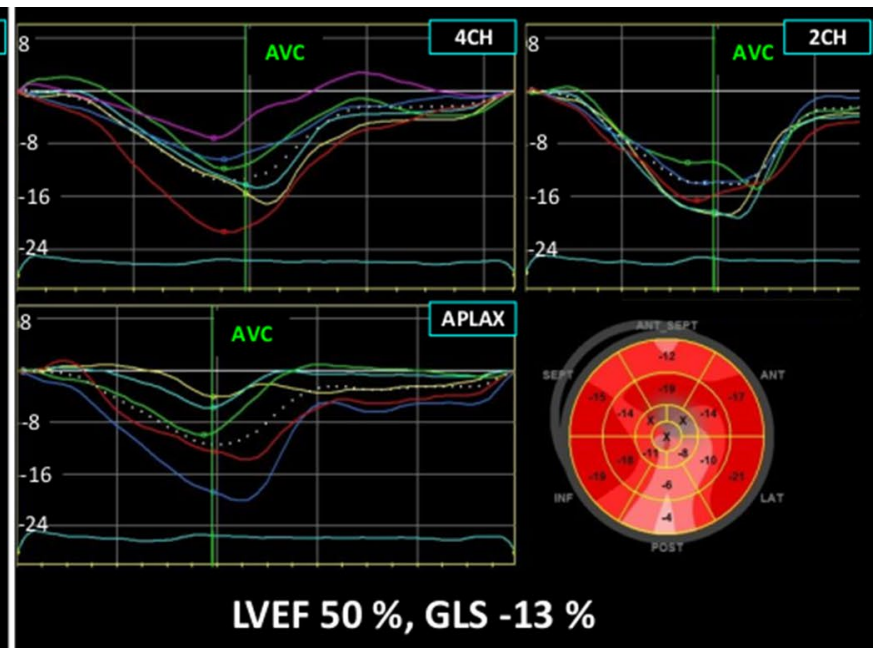

Fig. 3 Assessment of left ventricular (LV) function 1 month (left panel) and 3 months (right panel) after initiation of anthracycline therapy. Speckle tracking echocardiography shows impaired longitudinal function despite unchanged LV ejection fraction (EF)

time to uncover true cardiotoxicity. LVEF by 2DE suffers from low sensitivity in the detection of subclinical changes in cardiac function caused by early myocyte damage related to cancer treatment [28]. Given the higher temporal variability of $2 \mathrm{D} \mathrm{EF}$, the semi-automated $3 \mathrm{D} \mathrm{EF}$ with superior intraand interobserver and test-retest variability [3] seems to be the measurement of choice for serial evaluation of LVEF in patients at risk for CTRCD. Recognizing GLS as a clinically useful, feasible, and accurate measure of LV systolic function with ability to prognosticate subsequent CTRCD [29], the ASE/EACVI consensus statement accommodated a relative percentage decrease in GLS of $>15 \%$ from baseline as evidence of CTRD [27] (Fig. 3).

There is currently a paucity of data supporting strainguided strategy for initiation of cardioprotective treatment. However, the ongoing SUCCOUR multicenter trial will assess whether a strain-guided strategy could prevent subsequent cardiac dysfunction compared to the current EFguided management of patients receiving cardiotoxic cancer therapy [30].

\section{Future perspectives}

LVEF has a pivotal role in LV systolic assessment today, and will continue to be important in the future as long as therapeutic trials with LVEF as selection criteria remain the evidence base of societal guidelines. Nevertheless, the uncertainties of LVEF urge increased precision in systolic function estimation. 3D echocardiographic LVEF should be encouraged for serial monitoring of systolic function. More user-friendly and automated software for 3D LVEF and STE is expected to be announced allowing for wider application of more accurate techniques in less experienced hands. Evidence of STE-improved detection of systolic dysfunction and prognostification beyond volume-based methods is overwhelming, being particularly useful when volume-based methods signal systolic function in the low-normal or mildly impaired range. Insights will surely continue to emerge as we apply this technique to new cohorts, but will not bring STE into everyday cardiological practice unless new studies provide links to improved outcomes. Accordingly, diagnostic and therapeutic trials using GLS as selection criteria instead of LVEF are needed to change clinical practice.

\section{Conclusions}

Assessment of global systolic function is important for the management of all patients with cardiac diseases. Despite its conceptual and practical limitations, LVEF constitutes the evidence base of many of today's cardiology guidelines and is unlikely to be replaced in the near future. Given the uncertainties of LVEF estimations, the use of contemporary reliable measures of systolic function is needed. GLS by STE echocardiography is the best candidate to improve assessment of LV systolic function, but confirmation on ability to guide treatment decisions is still awaited.

\section{Compliance with ethical standards}

Conflict of interest Lars Gunnar Klaeboe and Thor Edvardsen declare that they have no conflicts of interest.

Open Access This article is distributed under the terms of the Creative Commons Attribution 4.0 International License (http://creativeco 
mmons.org/licenses/by/4.0/), which permits unrestricted use, distribution, and reproduction in any medium, provided you give appropriate credit to the original author(s) and the source, provide a link to the Creative Commons license, and indicate if changes were made.

\section{References}

1. Ponikowski P, Voors AA, Anker SD, et al. 2016 ESC Guidelines for the diagnosis and treatment of acute and chronic heart failure The Task Force for the diagnosis and treatment of acute and chronic heart failure of the European Society of Cardiology (ESC) Developed with the special contribution of the Heart Failure Association (HFA) of the ESC. Eur Heart J. 2016;37:2129-200.

2. Lang RM, Badano LP, Mor-Avi V, et al. Recommendations for cardiac chamber quantification by echocardiography in adults: an update from the american society of echocardiography and the european association of cardiovascular imaging. Eur Heart $\mathbf{J}$ Cardiovasc Imaging. 2015;16:233-71.

3. Thavendiranathan P, Grant AD, Negishi T, et al. Reproducibility of echocardiographic techniques for sequential assessment of left ventricular ejection fraction and volumes: application to patients undergoing cancer chemotherapy. J Am Coll Cardiol. 2013;61:77-84.

4. Stokke TM, Hasselberg NE, Smedsrud MK, et al. Geometry as a confounder when assessing ventricular systolic function: comparison between ejection fraction and strain. J Am Coll Cardiol. 2017;70:942-54.

5. Curtis JP, Sokol SI, Wang Y, et al. The association of left ventricular ejection fraction, mortality, and cause of death in stable outpatients with heart failure. J Am Coll Cardiol. 2003;42:736-42.

6. Cikes M, Solomon SD. Beyond ejection fraction: an integrative approach for assessment of cardiac structure and function in heart failure. Eur Heart J. 2016;37:1642-50.

7. Amundsen BH, Helle-Valle T, Edvardsen T, et al. Noninvasive myocardial strain measurement by speckle tracking echocardiography: validation against sonomicrometry and tagged magnetic resonance imaging. J Am Coll Cardiol. 2006;47:789-93.

8. Voigt JU, Pedrizzetti G, Lysyansky P, et al. Definitions for a common standard for 2D speckle tracking echocardiography: consensus document of the EACVI/ASE/Industry Task Force to standardize deformation imaging. Eur Heart J Cardiovasc Imaging. 2015;16:1-11.

9. Mirea O, Pagourelias ED, Duchenne J, et al. Intervendor differences in the accuracy of detecting regional functional abnormalities: a report from the EACVI-ASE strain standardization Task Force. JACC Cardiovasc Imaging. 2018;11:25-34.

10. Mirea O, Pagourelias ED, Duchenne J, et al. Variability and reproducibility of segmental longitudinal strain measurement: a report from the EACVI-ASE strain standardization task force. JACC Cardiovasc Imaging. 2018;11:15-24.

11. Stanton T, Leano R, Marwick TH. Prediction of all-cause mortality from global longitudinal speckle strain: comparison with ejection fraction and wall motion scoring. Circ Cardiovasc Imaging. 2009;2:356-64.

12. Ersboll M, Valeur N, Mogensen UM, et al. Prediction of all-cause mortality and heart failure admissions from global left ventricular longitudinal strain in patients with acute myocardial infarction and preserved left ventricular ejection fraction. J Am Coll Cardiol. 2013;61:2365-73.

13. Shah AM, Claggett B, Sweitzer NK, et al. The prognostic importance of impaired systolic function in heart failure with preserved ejection fraction and the impact of spironolactone. Circulation. 2015;132:402-14.
14. Liu H, Pozios I, Haileselassie B, et al. Role of global longitudinal strain in predicting outcomes in hypertrophic cardiomyopathy. Am J Cardiol. 2017;120:670-5.

15. Haugaa KH, Grenne BL, Eek CH, et al. Strain echocardiography improves risk prediction of ventricular arrhythmias after myocardial infarction. JACC Cardiovasc Imaging. 2013;6:841-50.

16. Elliott PM, Anastasakis A, Borger MA, et al. 2014 ESC guidelines on diagnosis and management of hypertrophic cardiomyopathy: the Task Force for the Diagnosis and Management of Hypertrophic Cardiomyopathy of the European Society of Cardiology (ESC). Eur Heart J. 2014;35:2733-79.

17. Pelliccia A, Caselli S, Sharma S, et al. European Association of Preventive Cardiology (EAPC) and European Association of Cardiovascular Imaging (EACVI) joint position statement: recommendations for the indication and interpretation of cardiovascular imaging in the evaluation of the athlete's heart. Eur Heart J. 2018;39:1949-69.

18. Liu D, Hu K, Niemann M, et al. Effect of combined systolic and diastolic functional parameter assessment for differentiation of cardiac amyloidosis from other causes of concentric left ventricular hypertrophy. Circ Cardiovasc Imaging. 2013;6:1066-72.

19. Holland DJ, Marwick TH, Haluska BA, et al. Subclinical LV dysfunction and 10-year outcomes in type 2 diabetes mellitus. Heart. 2015;101:1061-6.

20. Baumgartner H, Falk V, Bax JJ, et al. 2017 ESC/EACTS Guidelines for the management of valvular heart disease. Eur Heart J. 2017;38:2739-91.

21. Weidemann F, Herrmann S, Stork S, et al. Impact of myocardial fibrosis in patients with symptomatic severe aortic stenosis. Circulation. 2009;120:577-84.

22. Yingchoncharoen T, Gibby C, Rodriguez LL, et al. Association of myocardial deformation with outcome in asymptomatic aortic stenosis with normal ejection fraction. Circ Cardiovasc Imaging. 2012;5:719-25.

23. Kearney LG, Lu K, Ord M, et al. Global longitudinal strain is a strong independent predictor of all-cause mortality in patients with aortic stenosis. Eur Heart J Cardiovasc Imaging. 2012;13:827-33.

24. Smedsrud MK, Sarvari S, Haugaa KH, et al. Duration of myocardial early systolic lengthening predicts the presence of significant coronary artery disease. J Am Coll Cardiol. 2012;60:1086-93.

25. Eek C, Grenne B, Brunvand H, et al. Strain echocardiography predicts acute coronary occlusion in patients with non-ST-segment elevation acute coronary syndrome. Eur J Echocardiogr. 2010;11:501-8.

26. Antoni ML, Mollema SA, Delgado V, et al. Prognostic importance of strain and strain rate after acute myocardial infarction. Eur Heart J. 2010;31:1640-7.

27. Plana JC, Galderisi M, Barac A, et al. Expert consensus for multimodality imaging evaluation of adult patients during and after cancer therapy: a report from the American Society of Echocardiography and the European Association of Cardiovascular Imaging. Eur Heart J Cardiovasc Imaging. 2014;15:1063-93.

28. Kongbundansuk S, Hundley WG. Noninvasive imaging of cardiovascular injury related to the treatment of cancer. JACC Cardiovasc Imaging. 2014;7:824-38.

29. Thavendiranathan P, Poulin F, Lim K-D, et al. Use of Myocardial strain imaging by echocardiography for the early detection of cardiotoxicity in patients during and after cancer chemotherapy: a systematic review. J Am Coll Cardiol. 2014;63:2751-68.

30. Negishi T, Thavendiranathan P, Negishi K, et al. Rationale and design of the strain surveillance of chemotherapy for improving cardiovascular outcomes: the SUCCOUR trial. JACC Cardiovasc Imaging. 2018;11:1098-105. 